\title{
CYCLIC SUSPENSION OF KNOTS AND PERIODICITY OF SIGNATURE FOR SINGULARITIES ${ }^{1}$
}

\author{
BY WALTER D. NEUMANN
}

Communicated by Glen E. Bredon, March 8, 1974

By a knot we mean a pair $\left(S^{m}, M^{m-2}\right)$ with $M^{m-2}$ a smooth closed oriented submanifold of $S^{m}(m \geqq 3)$. If such a knot is given and $i: S^{m} \rightarrow$ $S^{m+2}$ is the standard embedding, then one can isotope $i$ in an essentially unique way (Lemma 1 below) to an embedding $j: S^{m} \rightarrow S^{m+2}$ whose intersection with $i S^{m}$ is $M \subset S^{m}$ transversally. The $n$-fold cyclic branched cover of $\left(S^{m+2}, i S^{m}\right)$ branched along $\left(j S^{m}, M^{m-2}\right)$ exists uniquely and is a manifold pair $\left(S_{n}^{m+2}, M_{n}^{m}\right)$, where $S_{n}^{m+2}$ is diffeomorphic to the sphere. This pair we call the $n$-fold cyclic suspension of $\left(S^{m}, M^{m-2}\right)$, or briefly $n$ suspension.

This construction is motivated by the following theorem. Recall that if $g:\left(C^{k}, 0\right) \rightarrow(C, 0)$ is a polynomial with isolated singularity at zero, the link $K_{g} \subset S^{2 k-1}$ of $g$ is the intersection of $g^{-1}(0)$ with a sufficiently small sphere $S^{2 k-1} \subset C^{k}$ at the origin.

THEOREM 1. If $g:\left(C^{k}, 0\right) \rightarrow(C, 0)$ is a polynomial with isolated singularity at zero, and $f:\left(C^{k+1}, 0\right) \rightarrow(C, 0)$ is the polynomial $f\left(z_{1}, \cdots, z_{k+1}\right)=$ $g\left(z_{1}, \cdots, z_{k}\right)+z_{k+1}^{n}$, then the link $\left(S^{2 k+1}, K_{f}\right)$ off at zero is diffeomorphic to the $n$-suspension of the link $\left(S^{2 k-1}, K_{g}\right)$ of $g$.

In particular we get a remarkably simple iterative topological construction of the Brieskorn manifolds [2] as repeated cyclic suspensions of torus links.

The above result has been announced independently by L. Kauffman [4] for weighted homogenious polynomials using an equivalent construction defined for knots whose complement $S^{m}-M^{m-2}$ fibres over $S^{\mathbf{1}}$ (fibred knots). Another version is due to Bredon [1] when $n=2$.

ADDED IN PROOF. The full construction and Theorem 1 have been found independently by Kauffman (private communication); a more general construction, which for links of isolated singularities of polynomials $f(x)$ and $g(y)$ gives the link for $f(x)+g(y)$, has also been found independently by Kauffman and the author.

AMS (MOS) subject classifications (1970). Primary 32C40, 57C54; Secondary 57A65.

Key words and phrases. Knot, isolated singularity, signature.

${ }^{1}$ Research partly supported by the Sonderforschungsbereich Theoretische Mathematik 40 in Bonn. 
For any knot $\left(S^{2 k+1}, M^{2 k-1}\right)$ the signature is defined (as the signature of a Seifert surface if $k$ is even or-up to sign-as the signature of the symmetrized Seifert linking form for abritrary $k)$. For the link $\left(S^{2 k+1}, K_{f}\right)$ of a singularity this is also called $\operatorname{sign}(f)$.

THEOREM 2. Let $\left(S^{2 k+1}, M_{n}^{2 k-1}\right), n=1,2, \cdots$, be the cyclic suspensions of a knot $\left(S^{2 k-1}, M^{2 k-3}\right)$ with $M^{2 k-3}(k-3)$-connected. Then there exists a unique $c \in \boldsymbol{R}$ such that

$$
\operatorname{sign}\left(S^{2 k+1}, M_{n}^{2 k-1}\right)=c n+p(n),
$$

where $p(n)$ is an almost periodic (hence bounded) function of $n$.

By "almost periodic" we mean the restriction of an almost periodic function (linear combination of periodic functions) from $\boldsymbol{R}$ to $\boldsymbol{R}$. If all the periods are rational, then $p(n)$ is of course genuinely periodic. Before describing a case where this happens, let me remark that the connectivity assumption in Theorem 2 can be greatly weakened and probably dropped. It suffices that the knot be bordant to a knot having a Seifert surface $F$ with $H_{k-2}(F ; Q)=0$. For a fibred knot whose fibre $F$ satisfies this condition, the data $c$ and $p(n)$ of Theorem 2 are calculated out of the monodromy $\mu: H_{k-1}(F) \rightarrow H_{k-1}(F)$, and the intersection form on this group. In fact if the eigenvalues of $\mu$ of unit length are $\exp \left(2 \pi i / q_{j}\right)\left(0<q_{j} \leqq 1\right.$, $j=1, \cdots, r)$, then $c$ and $p(n)$ only depend on the part of $H_{k-1}(F)$ belonging to these eigenvalues, and the $q_{j}$ are just the periods occurring in $p(n)$. In particular, the link of a singularity is such a fibered knot [5] and all the eigenvalues are roots of unity [3], so

COROLlary. If $f$ is as in Theorem 1, then $\operatorname{sign}(f)$, as a function of $n$, is of the form $c n+p(n)$ with $p(n)$ periodic of period the l.c.m. of the orders of the eigenvalues of the monodromy of $f$.

The existence of such a statement had been conjectured by Brieskorn, Durfee, and Zagier.

Proofs. We will show the proof of Theorem 1 in some detail. The existence and uniqueness of the embedding $j$ in the definition of cyclic suspension is given by the following lemma, which we deduce from Lemma 2 below.

LEMMA 1. Let $M^{m-2} \subset N^{m} \subset X^{m+2}$ be closed oriented manifolds and smooth embeddings with $N$ 2-connected. If $i: N \rightarrow X$ is the given embedding, there exists an embedding $j: N \rightarrow X$ such that

(a) $j$ is transversal to $i$ with intersection $M$;

(b) $j$ is isotopic to $i$ through embeddings satisfying (a).

Further, $j$ is uniquely defined by these properties up to isotopy through embeddings satisfying (a). 
Lemma 2. If $M^{m-2} \subset N^{m}$ are as in Lemma 1, then there exists a map $f: N \rightarrow D^{2}$ having zero as a regular value and $M=f^{-1}(0)$. Further $f$ is unique up to homotopy through maps with the same property.

To prove Lemma 2, note that its conclusion is equivalent to saying that there is a homotopy unique map $f_{0}: N-M \rightarrow S^{1}$ which restricts to a bundle trivialization $\partial U \rightarrow S^{1}$ of the boundary of a tubular neighbourhood of $M$ in $N$. Standard algebraic topology shows the existence and uniqueness to be equivalent, respectively, to: the dual class of $M$ in $H^{2}(N)$ is zero; $H^{1}(N)=0$. This proves Lemma 2. To prove Lemma $1:$ the existence of a trivialized tubular neighbourhood $t: N \times D^{2} \subset X$ of $N$ in $X$ is implied by $H^{2}(N)=0$. With $f$ as in Lemma 2, the map $j(x)=t(x, f(x))$ then satisfies (a) and (b). To see uniqueness, let $j_{1}$ be any map satisfying (a) and (b), and let $j_{s}, 0 \leqq s \leqq 1$, be the isotopy of (b). For $s>0$ sufficiently small, $j_{s}$ has the form $j_{s}=t\left(h_{s}(x), f_{s}(x)\right)$ with $f_{s}$ satisfying Lemma 2 , and $h_{s}$ a diffeomorphism of $N$ which is isotopic to the identity by $h_{r}, 0 \leqq r \leqq s$. The uniqueness thus follows from the uniqueness in Lemma 2.

Proof of Theorem 1. Let $f$ and $g$ be as in Theorem 1. Choose $\varepsilon>0$ and put

$$
S_{\varepsilon}^{2 k+1}=\left\{z \in C^{k+1} \mid\|z\|=\varepsilon\right\},
$$

and for $0 \leqq t \leqq 1$ put $S_{\varepsilon}(t)=\left\{z \in S_{\varepsilon}^{2 k+1} \mid \operatorname{tg}(z)+z_{k+1}=0\right\}$. For $\varepsilon$ small the $S_{\varepsilon}(t)$ are $(2 k-1)$-spheres and give an isotopy of the standard sphere $S_{\varepsilon}(0)=$ $S_{\varepsilon}^{2 k-1}$ to $S_{\varepsilon}(1)$. Also $S_{\varepsilon}(t) \cap S_{\varepsilon}(1)=K_{g}$ transversally for each $t<1$. Thus we can take $S_{\varepsilon}(1) \subset S_{\varepsilon}^{2 k+1}$ as our "standard embedding", and $S_{\varepsilon}(0) \subset S_{\varepsilon}^{2 k+1}$ as the embedding $j$ of Lemma 1 . If

$$
\bar{S}_{\varepsilon}^{2 k+1}=\left\{z \in C^{k+1} \mid\left\|z_{1}\right\|^{2}+\cdots+\left\|z_{k}\right\|^{2}+\left\|z_{k+1}\right\|^{2 n}=\varepsilon^{2}\right\}
$$

and

$$
\bar{K}_{f}=\bar{S}_{\varepsilon}^{2 k+1} \cap f^{-1}(0),
$$

then $\pi: \bar{S}_{\varepsilon}^{2 k+1} \rightarrow S_{\varepsilon}^{2 k+1}$ given by $\pi\left(z_{1}, \cdots, z_{k+1}\right)=\left(z_{1}, \cdots, z_{k+1}^{n}\right)$ gives a branched covering $\left(\bar{S}_{\varepsilon}^{2 k+1}, \bar{K}_{f}\right) \rightarrow\left(S_{\varepsilon}^{2 k+1}, S_{\varepsilon}(1)\right)$ branched along $\left(S_{\varepsilon}(0), K_{g}\right)$, and hence identifies $\left(\bar{S}_{\varepsilon}^{2 k+1}, \bar{K}_{f}\right)$ as the $n$-suspension of $\left(S_{\varepsilon}^{2 k-1}, K_{g}\right)$. It thus just remains to show that the "stertched" link $\left(\bar{S}_{\varepsilon}^{2 k+1}, \bar{K}_{f}\right)$ is diffeomorphic to $\left(S_{\varepsilon}^{2 k+1}, K_{f}\right)$. This is done by pushing the latter pair out to the "stretched" pair along a vector field defined on a small disc minus origin in $C^{k+1}-\{0\}$. Such a vector field $w$ can be obtained as follows: by a slight sharpening of Lemma 5.9 of Milnor [5] there is a vector field $v$ on a small disc minus origin in $C^{k}-\{0\}$ such that (in the notation of [5]) $\langle v(z)$, grad $\log g(z)\rangle=1$ for $g(z) \neq 0$ and $\langle v(z), z\rangle$ has positive real part. Then the vector field $w_{0}(z)=\left(v\left(z_{1}, \cdots, z_{k}\right),\left(z_{k+1} / n\right)\right)$ is suitable on $\boldsymbol{C}^{k} \times \boldsymbol{C}-\{0\} \times \boldsymbol{C}$, and $w_{1}(z)=$ $\left(0, z_{k+1}\right)$ is suitable in a thin neighbourhood of $\{0\} \times(C-\{0\})$, so pasting $w_{0}$ and $w_{1}$ with a partition of unity gives the required $w$. 
Proof of Theorem 2. First some fairly easy remarks.

(i) Iterated cyclic suspensions commute with each other.

(ii) Cyclic suspension preserves fibered structure of fibered knots.

(iii) Bordisms of knots can be cyclically suspended.

(iv) If $F^{m-1}$ is a Seifert surface of $\left(S^{m}, M^{m-2}\right)$, then a typical Seifert surface $F_{n}^{m+1}$ of the $n$-suspension is the $n$-fold branched cover of $D^{m+1}$ along a properly embedded $F^{m-1} \subset D^{m+1}$ obtained by pushing $F^{m-1} \subset$ $S^{m}=\partial D^{m+1}$ slightly into $D^{m+1}$.

Now given a knot $\left(S^{2 k-1}, M^{2 k-3}\right)$ as in Theorem 2 or the subsequent remarks, the fact that 2 -suspension preserves signature up to sign ([1], see also (v) below) and (i) above show we can assume $k$ even. Also we can assume we have a Seifert surface $F^{2 k-2}$ with $H_{k-2}(F ; Q)=0$, since (using (iv)) cyclic suspension preserves this property. This is enough to show (using C. T. C. Wall [6]) that cutting the branch locus $F$ out of $F_{n}$ in (iv) does not change the signature of $F_{n}$. We then have an unbranched covering and Theorem 2 becomes a special case of the following, which will be discussed in detail elsewhere.

THEOREM. If $X^{4 r}$ is a compact manifold with boundary and, $a: X \rightarrow S^{1}$ is a map, let $X_{n} \rightarrow X$ be the induced cover from the $n$-fold cyclic cover $S^{1} \rightarrow S^{1}$. Then as a function of $n, \operatorname{sign}\left(X_{n}\right)=c n+p(n)$ with $c$ constant and $p(n)$ almost periodic. Further, if a $\mid \partial X$ is a fibration, then the periods of $p(n)$ are $q_{j}$ $(j=1, \cdots, r)$ where $\exp \left(2 \pi i / q_{j}\right)$ are the eigenvalues of unit length of the middle dimensional monodromy of a $\mid \partial X$. (Actually in general $p(n)$ and $c-$ $\operatorname{sign}(X)$ are homotopy invariants of $(\partial X, a \mid \partial X)$ and are calculated out of $a$ generalized "monodromy" analogous to monodromy of a fibration.)

Finally, an alternative proof of Theorem 2-I do not know how feasible-is suggested by the fact:

(v) $n$-suspension tensors the Seifert linking form of $\left(S^{2 k-1}, M^{2 k-3}\right)$ by the $(n-1)$-square matrix (up to sign)

$$
\left(\begin{array}{cccccc}
1 & 1 & 0 & \cdot & \cdot & 0 \\
0 & 1 & 1 & \cdot & \cdot & 0 \\
\cdot & & & & \cdot \\
\cdot & & & & \cdot \\
0 & \cdot & \cdot & \cdot & 1 & 1 \\
0 & \cdot & \cdot & \cdot & 0 & 1
\end{array}\right)
$$

This has also been shown by Kauffman for fibered knots, and by Bredon for $n=2$.

\section{BIBLIOGRAPHY}

1. Glen E. Bredon, Regular $O(n)$ manifolds, suspension of knots, and knot periodicity, Bull. Amer. Math. Soc. 79 (1973), 87-91. MR 46 \#9999. 
2. E. Brieskorn, Beispiele zur Differenzialtopologie von Singularitäten, Invent. Math. 2 (1966), 1-14. MR 34 \#6788.

3. - Die Monodromie der isolierten Singularitäten von Hyperflächen, Manuscripta Math. 2 (1970), 103-161. MR 42 \#2509.

4. Louis H. Kauffman, Branched coverings, open books and knot periodicity, Preprint and Notices Amer. Math. Soc. 20 (1973), 619. Abstract \#708-G7.

5. J. Milnor, Singular points of complex hypersurfaces, Ann. of Math. Studies, no. 61, Princeton Univ. Press, Princeton, N.J.; Univ. of Tokyo Press, Tokyo, 1968. MR 39 \#969.

6. C. T. C. Wall, Non-additivity of the signature, Invent. Math. 7 (1969), 269-274. MR 39 \#7615.

Mathematisches Institut, Universität BonN, 53 BonN, GeRmany 